\title{
Alveolar Soft Part Sarcoma of the Tongue in an Adolescent
}

\author{
Amaranto Suárez Sr. ${ }^{1}$, Kelly Paz ${ }^{1}$, Mario Melo-Uribe ${ }^{2}$, Andrey Moreno ${ }^{3}$, Alejandra Calderon ${ }^{1}$ \\ 1. Pediatric Oncology, Instituto Nacional de Cancerología, Bogotá, COL 2. Pathology, Instituto Nacional de \\ Cancerología, Bogotá, COL 3. Head and Neck Surgery, Instituto Nacional de Cancerología, Bogotá, COL
}

Corresponding author: Alejandra Calderon, calderongasca@gmail.com

\begin{abstract}
Alveolar soft part sarcoma is a rare malignant soft tissue neoplasm of uncertain histogenesis and aggressive clinical behavior. Alveolar soft part sarcoma arises in the head and neck in $27 \%$ of cases, with $25 \%$ of head and neck cases occurring in the tongue. Herein the case of a pediatric patient diagnosed with alveolar soft tissue sarcoma of the tongue, who received surgical treatment with total resection of the lesion and chemotherapy without radiotherapy, is presented. To date, the patient is in remission of the disease.
\end{abstract}

Categories: Pediatrics, Pediatric Surgery, Oncology

Keywords: alveolar soft part sarcoma, tongue, children, non-rhabdomyosarcoma soft tissue sarcoma

\section{Introduction}

Alveolar soft part sarcoma (ASPS) is a rare malignant neoplasm of uncertain histogenesis, first described in 1952 by Christopherson [1,2,3]. It represents between $0.4 \%$ and $1.0 \%$ of all soft tissue sarcomas and occurs in $5 \%$ of all non-rhabdomyosarcoma soft tissue sarcomas in pediatrics [4,5]. It predominantly affects adolescents and young adults between 15 and 35 years of age with a predominance in women, with an H:M ratio of $1: 1.4[6,7]$.

In adults, the most common location is the lower extremities' deep soft tissues (thighs and buttocks) in contrasts with the presentation in children and adolescents where there is a predilection for the location in the head and neck, in particular, the tongue (where it occurs in approximately $25 \%$ of cases) and the orbit $[8,9,10]$.

Review began 10/14/2020 Review ended 11/03/2020 Published 11/16/2020

() Copyright 2020

Suárez et al. This is an open access article distributed under the terms of the Creative Commons Attribution License CC-BY 4.0., which permits unrestricted use, distribution, and reproduction in any medium, provided the original author and source are credited.
Clinically indolent, it presents as a painless, slow-growing mass with great vascularity; the limited symptoms generate delays in diagnosis, which could explain that $80 \%$ of adult patients and $37 \%$ of pediatric patients present metastases at diagnosis [11]. The disease has a survival rate of $77 \%$ at two years, $60 \%$ at five years, $38 \%$ at 10 years, and $15 \%$ at 20 years [11]. In its localized form of presentation, five-year survival is $71 \%$; in metastatic disease, it is only $20 \%$ [11].

Alveolar soft part sarcoma of the tongue is a highly vascular tumor; diagnostic studies such as ultrasound can misdiagnose this tumor and be mistaken for benign lesions; so magnetic resonance imaging is the study of choice $[10,11]$. The initial diagnostic suspicion of alveolar soft part sarcoma of the tongue is complicated, causing delays in referral to specialized centers where the diagnosis is established $[10,11]$.

\section{Case Presentation}

A 13-year-old adolescent woman presented with a 14-month history of a non-ulcerated, painless, and lobulated mass on the left lateral ventral surface of the tongue, slow-growing, without other associated symptoms (Figure 1). 


\section{Cureus}

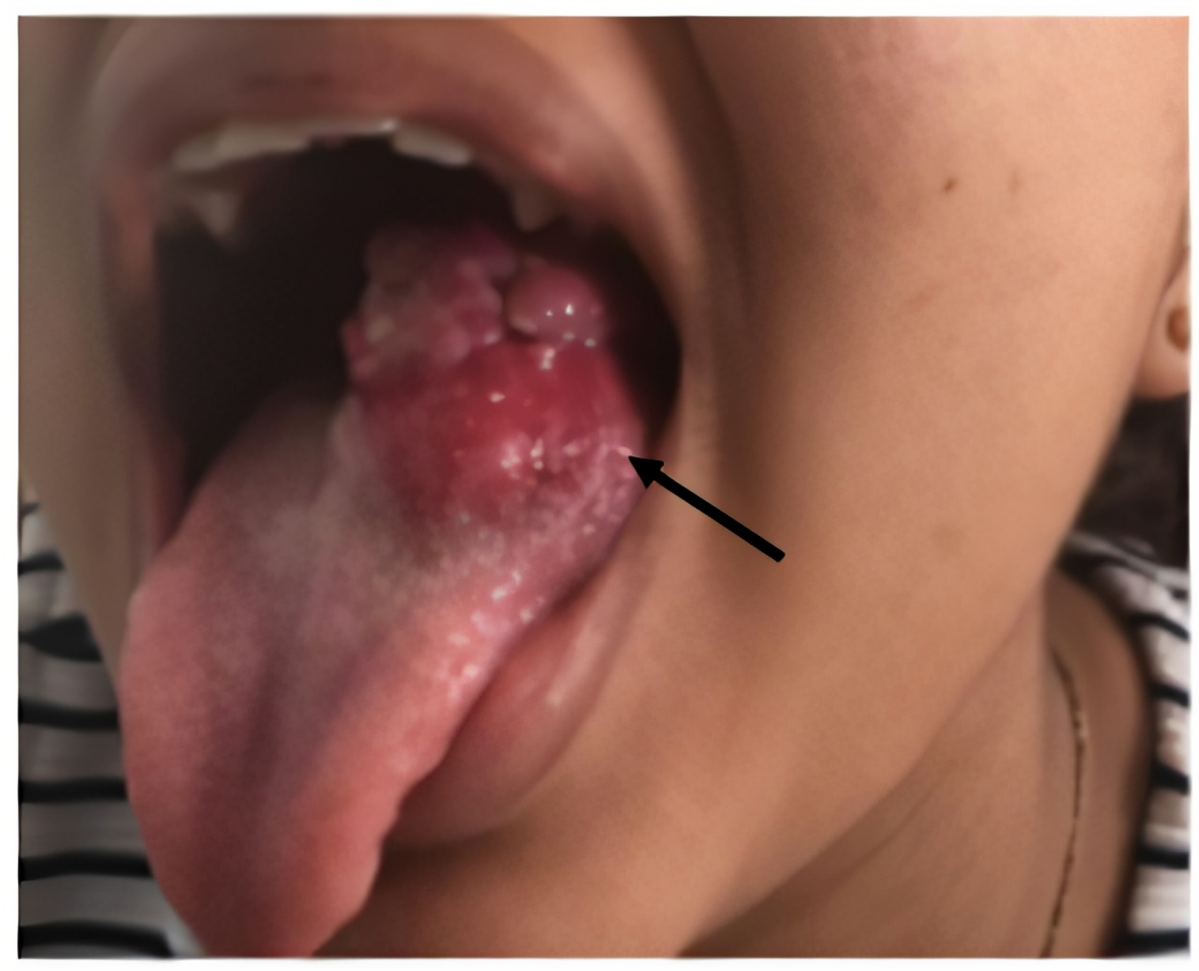

\section{FIGURE 1: Tongue mass.}

Non-ulcerated, painless, and lobulated mass on the left lateral ventral surface of the tongue,

A nuclear magnetic resonance imaging of the face and neck was performed, which showed a mass of welldefined and regular contours in the superior longitudinal, vertical, and transverse muscles of the lateral dorsum of the left hemitongue (Figure 2). 


\section{Cureus}

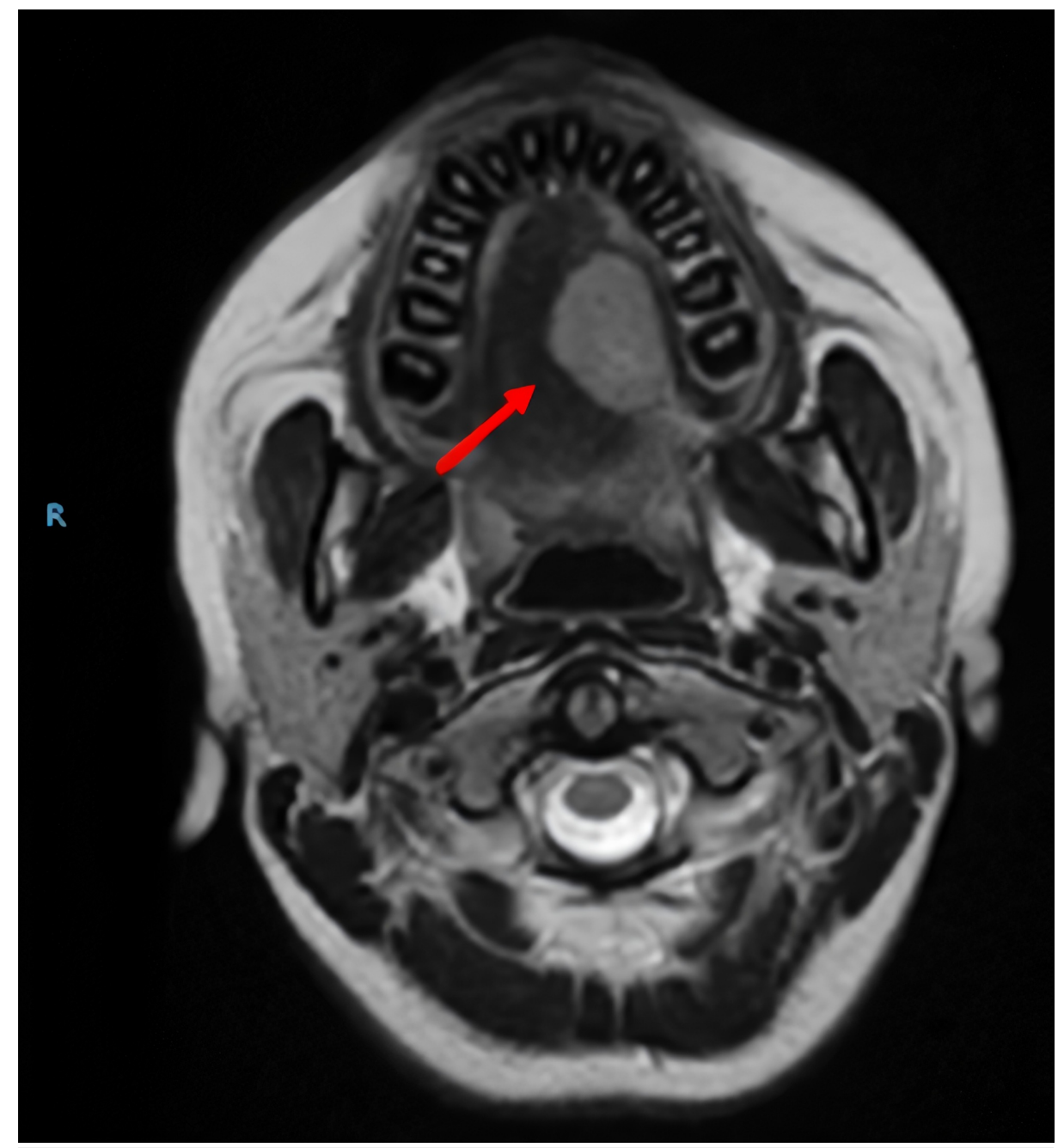

FIGURE 2: Magnetic resonance imaging of the face and neck.

Involvement of the mucosa and the superior longitudinal, vertical, and transverse muscles of the lateral dorsum of the left hemilanguage was observed by a mass of well-defined and regular contours with increased intensity of signal in the T2-weighted sequences, with intense and homogeneous enhancement with the contrast medium, with measurements of $21 \mathrm{~mm} \times 26 \mathrm{~mm} \times 21 \mathrm{~mm}$.

With a probable diagnosis of the tongue's hemangioma, resection was performed in a center outside ours. The pathology report showed a lesion compatible with an alveolar soft part sarcoma with one of the resection edges in contact with the tumor (Figure 3).

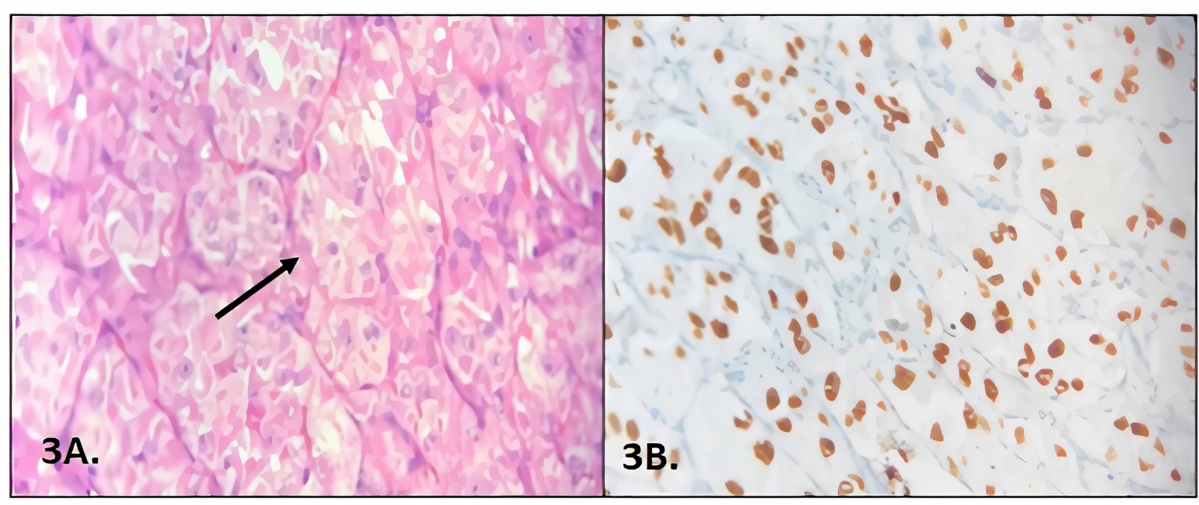

\section{FIGURE 3: Pathology and immunohistochemistry.}

3A: Lesion of well-defined nests of polygonal cells, eosinophilic granular cytoplasm, surrounded by thin septa fibrous, giving the appearance of alveoli formation, compatible with alveolar soft tissue sarcoma. 


\section{Cureus}

3B: Transcription factor E3 (TFE3) positive.

Taking into account the pathology report, upon admission to our institution, a second surgery was proposed to widen the surgical margins and lymph node dissection. Considering the presence of one of the edges in contact with the tumor and the initial non-oncological resection, the tumor was staged as Group-III of the Intergroup Rhabdomyosarcoma Study Group (IRSG) staging system [12].

The initiation of chemotherapy was indicated; three cycles of ifosfamide and doxorubicin were administered every 21 days. Subsequently, surgical resection was performed to widen the edges. The pathology is reported without residual tumor, with negative resection borders.

Three cycles of adjuvant chemotherapy were applied with the same agents, without radiotherapy. To date, the patient has completed treatment and is under follow-up, disease-free, and asymptomatic. Posttreatment MRI shows the scar where the tumor was, with no signs of tumor relapse (Figure 4).

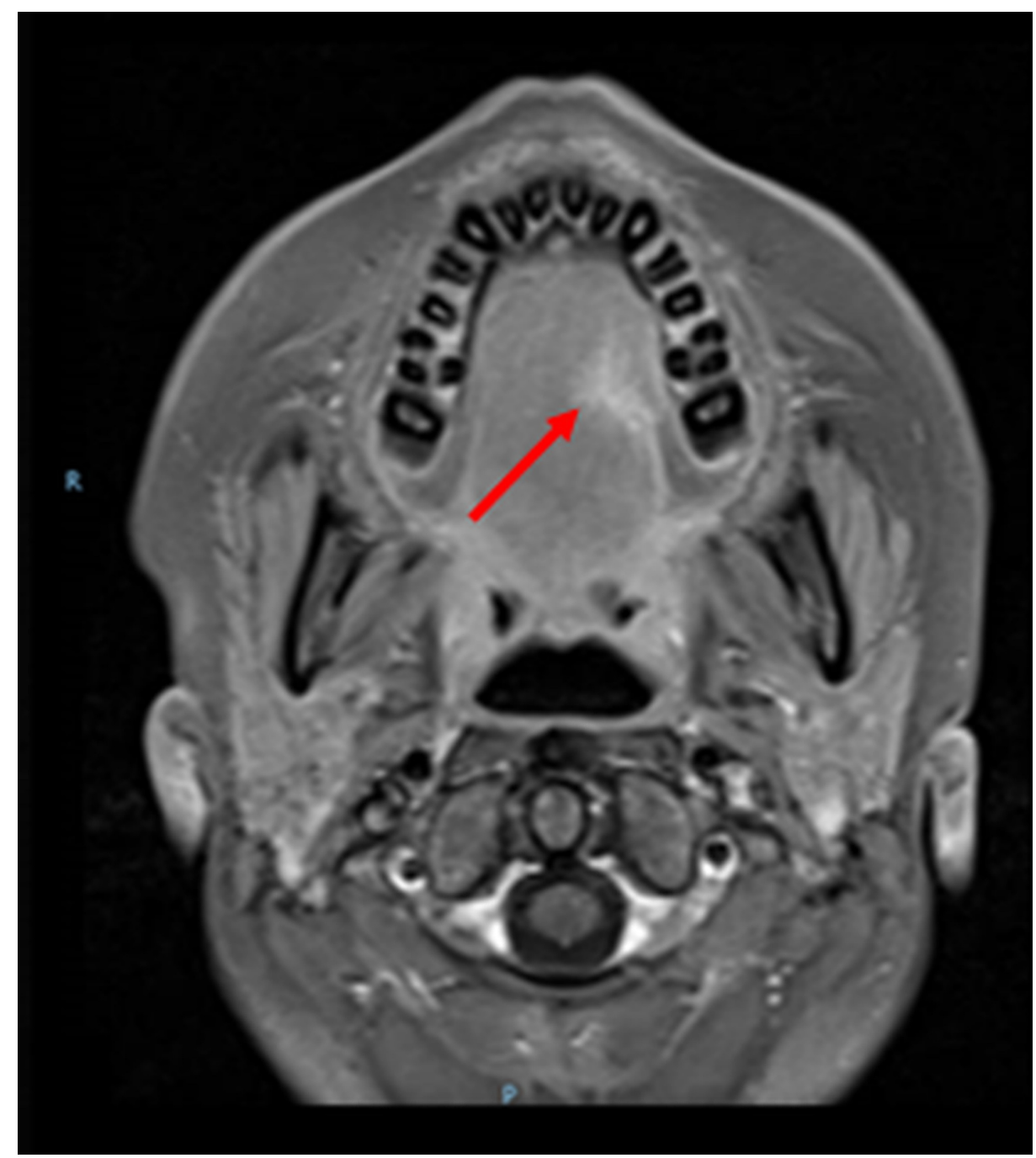

\section{FIGURE 4: Post-treatment MRI.}

Post-treatment MRI shows the scar where the tumor was, with no signs of tumor relapse.

\section{Discussion}

Soft part sarcoma is a rare neoplasm in pediatrics, accounting for $5 \%$ of all cases of non-rhabdomyosarcoma soft part sarcomas in pediatrics [4]. Despite being an indolent growth neoplasm, alveolar soft part sarcoma has a high propensity to metastasize, especially to the lung in up to $42 \%-65 \%$ of cases, with a metastatic presentation to the bones and brain being less common, even in early stages of the disease $[11,12]$. 
The five-year survival rate for alveolar soft tissue sarcoma in children, adolescents, and adults younger than 25 years is $83 \%$ [13]. However, due to the proclivity for late metastases, survival rates may decrease [13]. In the previously illustrated case, despite the time elapsed between the biopsy and the moment when negative resection margins were achieved, the patient did not present metastatic involvement.

In contrast to what was reported by Shelke et al. [11], who reported that the most frequent location of lingual alveolar sarcoma is the base of the tongue (18\%), followed by $13 \%$ on the back, $6 \%$ on the ventral surface, and $3 \%$ on the left lateral back [11], the patient presented the tumor on the ventral and left lateral surface of the tongue.

The clinical course was indolent as described in the literature as a hemangioma of the tongue for which a surgery limited to tumor resection with a positive border was performed, which led to an enlargement of the margins and lymph node dissection. In the presence of a non-metastatic tumor and in a location where radical resections leave significant morbidity in the soft tissues of the neck and long-term cosmetic and psychological side effects, conservative management was carried out with neoadjuvant, adjuvant chemotherapy, and surgery. At the end of treatment, the patient is in remission, free of signs of tumor recurrence or relapse.

\section{Conclusions}

It will always be important to take a detailed clinical history to reach the correct diagnosis of the soft part sarcoma of the tongue. A wrong diagnosis can lead to delay in the initiation of adequate treatment of the patient. The presentation of the disease in children, adolescents, and young adults makes us reflect on the need to form multidisciplinary groups with the active participation of adult clinical oncologists, head and neck surgeons, pathologists, radiation therapists, and rehabilitators.

\section{Additional Information \\ Disclosures}

Human subjects: Consent was obtained by all participants in this study. Conflicts of interest: In compliance with the ICMJE uniform disclosure form, all authors declare the following: Payment/services info: All authors have declared that no financial support was received from any organization for the submitted work. Financial relationships: All authors have declared that they have no financial relationships at present or within the previous three years with any organizations that might have an interest in the submitted work. Other relationships: All authors have declared that there are no other relationships or activities that could appear to have influenced the submitted work.

\section{References}

1. Christopherson WM, Foote FW, Stewart FW: Alveolar soft-part sarcomas; structurally characteristic tumors of uncertain histogenesis. Cancer. 1952, 5:1-100. 10.1002/1097-0142(195201)5:1<100::AIDCNCR2820050112>3.0.CO;2-K

2. Kinger M, Chakrabarti P, Varma A, Doshi B: Alveolar soft part sarcoma of tongue in 14-year-old boy . Ann Maxillofac Surg. 2014, 4:240-242. 10.4103/2231-0746.147165

3. Correia-Silvaa JF, Duartea ECB, Lacerda JCT, de Sousa SCOM, Mesquita RA, Gomeza RS: Alveolar soft part sarcoma of the tongue. Oral Oncology Extra. 2006, 42:241-243. 10.1016/j.ooe.2006.02.001

4. Noussios G, Chouridis P, Petropoulos L, Karagiannidis K, Kontzoglou G: Alveolar soft part sarcoma of the tongue in a 3-year-old boy: a case report. J Med Case Rep. 2010, 4:130. 10.1186/1752-1947-4-130

5. King VVB, Fee WE: Alveolar soft part sarcoma of the tongue. Am J Otolaryngol. 1983, 4:363-366. 10.1016/s0196-0709(83)80023-4

6. Charrier JB, Esnault O, Brette MD, Monteil JP: Alveolar soft-partsarcoma of the cheek. Br J Oral Maxillofac Surg. 2001, 39:394-397. 10.1054/bjom.2000.0635

7. Yigitbasi OG, Guney E, Kontas O, Somdas MA, Patiroglu T: Alveolar soft part sarcoma: report of a case occurring in the sinonasal region. Int J Pediatr Otorhinolaryngol. 2004, 68:1333-1337. 10.1016/j.ijporl.2004.04.020

8. Aiken A, Stone J: Alveolar soft-part sarcoma of the tongue. Am J Neuroradiol. 2003, 24:1156-1158.

9. Ordóñez NG, Mackay B: Alveolar soft-part sarcoma: a review of the pathology and histogenesis . Ultrastruct Pathol. 1998, 22:275-292. 10.3109/01913129809103349

10. Tekin B, Mehmet K, Cengiz D, et al.: Alveolar soft part sarcoma of the tongue . J Craniofac Surg. 2009, 20:2160-2162. 10.1097/SCS.0b013e3181bf0131

11. Shelke P, Sarode GS, Sarode SC, Anand R, Prajapati G, Patil S: Alveolar soft-part sarcoma of the oral cavity: a review of literature. Rare Tumors. 2018, 10:1-8. 10.1177/2036361318810907

12. Argyris PP, Reed RC, Manivel JC, et al.: Oral alveolar soft part sarcoma in childhood and adolescence: report of two cases and review of literature. Head Neck Pathol. 2013, 7:40-49. 10.1007/s12105-012-0395-y

13. Kayton ML, Meyers P, Wexler LH, Gerald WL, Laquaglia MP: Clinical presentation, treatment, and outcome of alveolar soft part sarcoma in children, adolescents, and young adults. J Pediatr Surg. 2006, 41:187-193. 10.1016/j.jpedsurg.2005.10.023 